\title{
Health Literacy and Shared Decision-making: Exploring the Relationship to Enable Meaningful Patient Engagement in Healthcare
}

\author{
Danielle M. Muscat, $P h D^{7}$ (D), Heather L. Shepherd, $P h D^{2}$, Don Nutbeam, $P h D^{3}$, \\ Lyndal Trevena, $P h D^{3}$, and Kirsten J. McCaffery, $P h D^{1,4}$
}

\begin{abstract}
${ }^{1}$ Faculty of Medicine and Health, School of Public Health, Sydney Health Literacy Lab, The University of Sydney Sydney, NSW, Australia; ${ }^{2}$ Faculty of Science, School of Psychology, The University of SydneySydney, NSW, Australia; ${ }^{3}$ Faculty of Medicine and Health, School of Public Health, The University of SydneySydney, NSW, Australia; ${ }^{4}$ Faculty of Medicine and Health, School of Public Health, Wiser Healthcare, The University of SydneySydney, NSW, Australia.
\end{abstract}

\begin{abstract}
Research into health literacy and shared decision-making has largely developed along parallel, but distinct lines over the past two decades. There is little evidence that the concepts and related practice have intersected except in the most functional way, for example, to simplify shared decision-making tools by improving readability scores of decision aids. This paper presents an integrated model to strengthen and sustain patient engagement in health care by drawing on the strengths of both concepts. This includes addressing patients' skills and capacities, alongside modifications to written and verbal information. We propose an expanded model of shared decision-making which incorporates health literacy concepts and promotes two-tiered intervention methods to improve the targeting and personalization of communication and support the development of transferable health literacy skills among patients.
\end{abstract}

KEY WORDS: shared decision-making; health literacy; model; literacy; communication; patient participation.

J Gen Intern Med 36(2):521-4

DOI: $10.1007 / \mathrm{s} 11606-020-05912-0$

(c) Society of General Internal Medicine 2020

\section{HEALTH LITERACY}

Since research and scholarly enquiry into health literacy began 25 years ago, the concept of health literacy has evolved and related research has developed substantially. ${ }^{1}$ Although numerous definitions of health literacy exist, ${ }^{2}$ almost all definitions in common use have the same core elements describing the personal skills and environmental conditions that enable individuals to obtain, understand, and use information to make decisions and take actions that will have an impact on health status. ${ }^{3}$ Despite this consistency across definitions, there remain differences in how health literacy is understood in relation to health outcomes.

On one hand, health literacy has been conceptualized as a risk factor for good health. ${ }^{4}$ This interest is observable in

Received February 27, 2020

Accepted May 4, 2020

Published online May 29, 2020 research into the measurement of health-related literacy, examination of the relationship between low literacy and a range of health conditions, and progressive testing of interventions designed to mitigate the effects of low literacy by removing literacy-related barriers and making information easier to understand and act on. ${ }^{4}$ There is clear and consistent evidence identifying the common gap between the literacy requirements of health information materials, and the literacy skills of the intended target audience. ${ }^{5}$

Health literacy is also conceptualized as a personal asset; a set of personal, transferable skills that can be developed to support greater independence in health decision-making through structured exposure to information that is targeted and personalized. ${ }^{4}$ Past research has distinguished a threelevel hierarchy of health literacy skills, ${ }^{6}$ described here. Functional health literacy refers to the basic reading and writing skills to obtain health information and apply knowledge to a limited range of prescribed activities and everyday situations. Communicative health literacy skills are those which can be used to participate actively in everyday situations, extract health information and derive meaning from different forms of health communication, and apply this to changing circumstances. Critical health literacy refers to the cognitive and social skills needed to critically assess the applicability of health information to personal situations or its reliability, and use this information to exert greater control over life events and situations.

Such a continuum positions health literacy as a modifiable determinant of health that is subject to change and improvement, and suggests that the different levels of health literacy progressively allow for greater autonomy in decision-making and personal empowerment. ${ }^{6}$ Edwards and colleagues' Health Literacy Pathway Model similarly highlights how patients transition along a "health literacy pathway" as they increase their knowledge and understanding of health conditions, learn how to manage them, and actively participate in discussions with healthcare professionals. ${ }^{7}$

This distinction between "risk-based" and "asset-based" models of health literacy provides an important starting point 
for understanding the relationship between health literacy and shared decision-making.

\section{UNDERSTANDING THE RELATIONSHIP BETWEEN SHARED DECISION-MAKING AND HEALTH LITERACY}

Shared decision-making is a process in which healthcare professionals and patients work together to select tests, treatments, management, or support packages, based on clinical evidence and the patient's values and informed preferences. ${ }^{8}$ As a midpoint between "paternalistic" and "consumerist" models of clinical decision-making, this approach seeks to recognize the autonomy and responsibility of both health professionals and patients [9].

In the field of shared decision-making, low health literacy has most often been positioned as a potential risk factor, and actions in relation to health literacy have tended to be goaldirected, seeking to mitigate the related risks of lower health literacy to achieve improved clinical outcomes. ${ }^{9}$ A seminal review of patient decision aids published in 2010 found that they were rarely developed with lower literacy populations in mind, and that measures of patient health literacy and decision aid readability were rarely reported. ${ }^{10}$ Since then, there have been increased efforts to reduce "risk" by removing literacyrelated barriers in decision aids. These include decreasing readability scores (e.g., $\left.{ }^{11}\right)$, using techniques to reduce cognitive effort (e.g., ${ }^{12}$ ), and including patients of all literacy levels in the development process (e.g., ${ }^{13}$ ). Alternative formats for decision support, such as Option Grids,${ }^{14}$ have also been developed which generally have less textual information compared with typical decision aids and are designed to be used jointly with a provider within an encounter.

Simplifying information is necessary but not sufficient to achieve active participation in decision-making. ${ }^{15}$ In essence, it represents a response from the healthcare provider to potential shortcomings in patients' ability to understand, engage, and apply information. However, for patients to successfully share in decision-making, they need skills to communicate effectively, to obtain, understand, and share information with health professionals (including, for example, alternative options, risks, benefits, and uncertainties). ${ }^{8,9,16,17}$ Patients also need the cognitive and social skills to express personal values, preferences, and experiences ${ }^{9,} 18$ and to contextualize and critically evaluate information to make a decision which aligns with these values and preferences. ${ }^{8}{ }^{19}$ These skills align with Nutbeam's three-level hierarchy of health literacy skills, ${ }^{6}$ described above. In fact, research has shown that, at different times in the shared decision-making process, all levels of health literacy skills (functional, communicative, and critical) are required for engagement with healthcare professionals. ${ }^{20}$ Research also shows that health literacy skills for shared decision-making are subject to change and improvement through education and training. ${ }^{19}$

\section{IMPLICATIONS FOR RESEARCH AND PRACTICE}

Understanding health literacy from the perspective of both patient (transferable skills) and provider (improved communication) invites a close examination of existing models for shared decision-making. Hoffmann et al.'s 2014 model of shared decision-making presented in Figure 1, for example, has a strong provider focus. The authors clearly articulate that providers need to find and appraise research evidence and practice patient-centered communication to support shared decision-making within consultations. ${ }^{21}$ The implications are necessarily provider-focused. They include calls to integrate shared decision-making and evidence-based medicine in medical education and training and bring these concepts together in the development and implementation of clinical practice guidelines. ${ }^{21}$

What this model misses, however, is a recognition of the complementary skills that it seems patients are assumed to have to "incorporate the evidence and expertise of the clinician, along with their values and preferences, into their decision-making". ${ }^{21}$ After all, it does take "two to tango". ${ }^{22}$ Extending this model to include a patient health literacy skills component (Fig. 1) recognizes that patients require skills to participate in the shared decision-making process in much the same way that providers need skills to engage with their patients. ${ }^{23-26}$ This includes skills to obtain, understand, and use evidence-based information about the natural history of the condition, the possible options, the benefits and harms of each, and a quantification of these, as well as the ability to deliberate on and articulate their values to construct informed preferences.

This expanded model points to several necessary shifts in our practice to support meaningful patient engagement. First, two-tiered intervention approaches are needed. This includes interventions to support the continued targeting and personalization of written resources for shared decision-making based on best-practices in health literacy, ${ }^{9,27}$ as well as developing interventions oriented towards building health literacy skills among patients. Regarding the former, evidence from systematic reviews suggests that several discrete design features can improve participant comprehension. These include reducing the amount of text and medical jargon ${ }^{28}$; presenting essential information by itself or first; presenting numerical information in logical ways, for example, so that the higher number is better; and adding video to verbal narrative. ${ }^{27}$ Work is also continuing to build the evidence base for the impact of images as an effective way to communicate health information. ${ }^{29}$

Considering interventions oriented towards building health literacy skills among patients, currently, most shared decisionmaking interventions such as decision aids and decision coaching tend to focus on contextually specific information and skills which are required for a specific decision. This undoubtedly helps patients to obtain health information and apply new knowledge to a limited range of prescribed activities (i.e., functional health literacy). However, more 
Original model:

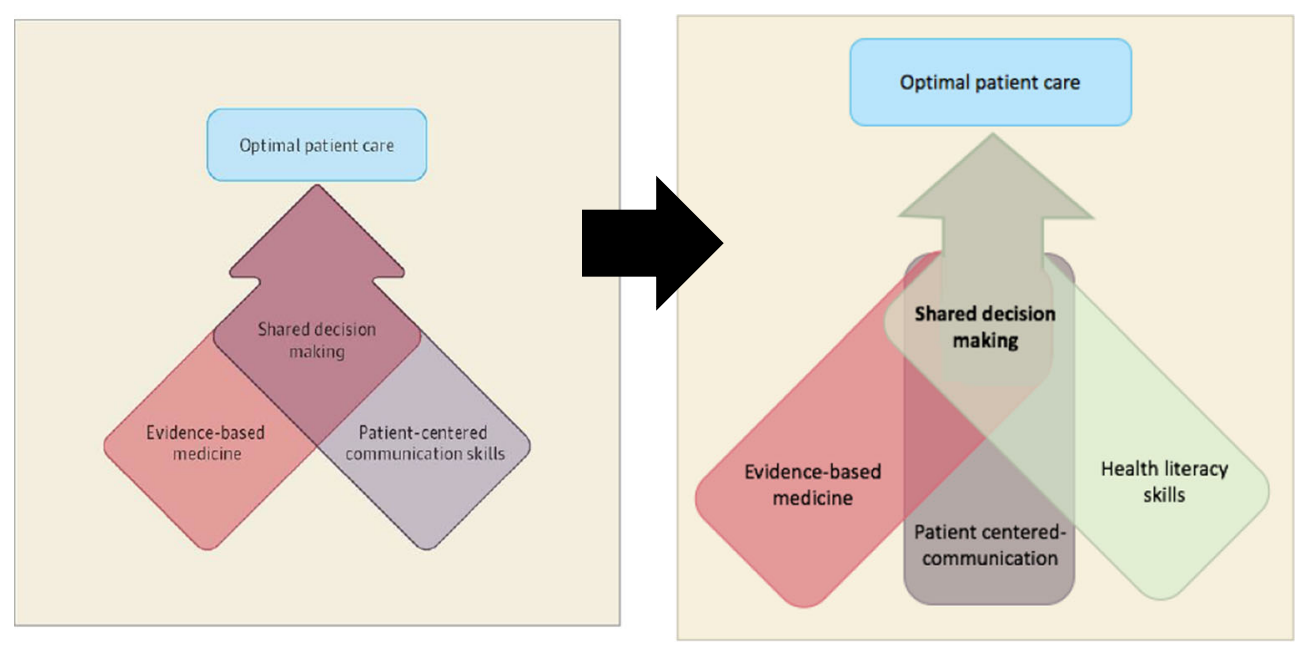

Fig. 1 Expanded model of shared decision-making, accounting for patients' health literacy skills. Adapted from Hoffmann et al. ${ }^{22}$

transferable skills (associated with communicative and critical health literacy) are also needed to achieve the goal of empowerment and support greater autonomy across health decisions. Of course, patients need context-specific skills and knowledge to support shared decision-making about specific health conditions. Knowledge about diabetes and skills to interpret $\mathrm{HbA1c}$ levels, for example, are necessary to support informed preferences and decision-making about diabetes management. Understanding the specific risks and benefits associated with different treatment options for end-stage kidney disease is necessary to make an informed choice about kidney replacement therapies or conservative care.

However, interventions are also needed to develop patients' basic core health literacy skills which can be applied across decision-making contexts. Examples of such skills include social and cognitive skills to ask questions; deliberate on and share values and preferences; and understand information about evidence, options, outcomes, and probabilities (including numeric information). ${ }^{30}$ As these skills are transferable, interventions which address them are more likely to lead to meaningful and sustained shared decision-making for patients over time. For example, a patient with comorbid kidney disease and diabetes can use generic skills to formulate and ask questions about treatment options to make a shared decision about hemo- or peritoneal dialysis with their nephrologist and then again to share in decision-making about medication options with their endocrinologist at a later date.

Such an approach often requires more time and can best be delivered in a more structured educational setting. This might be through established Adult Basic Education programs, schools, or health educational settings like diabetes educator group sessions. Well-designed on-line learning programs have also been proposed as a platform to support skill-development. ${ }^{31}$ While these avenues have been largely unexplored, encouraging signs are emerging. This includes through the development and evaluation of the Informed Health Choices primary school intervention to improve the skills and ability of children to assess the reliability of claims about treatment effects across clinical contexts, ${ }^{32}$ as well as a generic shared decision-making training program implemented in adult basic education settings in Australia. ${ }^{19,}{ }^{30}$ Examples of health literacy skills for shared decision-making addressed in these programs include the following: basic literacy (reading and writing) skills to understand shared decision-making terminology (e.g., options, benefits, harms); numerical literacy skills to understand and compare risk information; graphical literacy skills to interpret icon arrays ${ }^{19,30}$; and critical skills to recognize when a claim about the effects of treatments has an untrustworthy basis. ${ }^{32}$ In addition to continuing and expanding this work in these structured settings, there is also a need for research into opportunistic skills development that could be integrated within healthcare delivery.

The second shift in practice suggested by our expanded model regards the integration of health literacy into shared decision-making training programs for health professionals (and/or shared decision-making into health literacy training). To date, training in health literacy and shared decision-making has tended to occur in silos both in initial education and as a part of continuing professional development, with only recent signs of integration (see, for example, ${ }^{33}$ ). Bringing training together can foster greater appreciation of how efforts to support shared decision-making need to acknowledge health literacy and recognition of the implications of addressing health literacy on patient engagement in decision-making.

\section{CONCLUSION}

We have examined the alternative ways of conceptualizing health literacy and considered practical intervention approaches to develop health literacy skills for shared decisionmaking. We recognize that a patient's level of involvement in decision-making is a personal preference. Eliciting and 
respecting patients' choice to be involved in decision-making (or not) are, therefore, important but should occur in a context in which patients understand what involvement means, recognize that there are no right or wrong decisions in situations of equipoise, and appreciate that they have a valuable contribution to make given the complementary knowledge that they bring to a healthcare encounter (e.g., past experiences, values, and preferences). ${ }^{34}$ By supporting the development of generic health literacy skills for shared decision-making, patients will have a transferable skill-set should they wish to use it.

By positioning health literacy as an asset for shared decision-making, we can begin to recognize previously unidentified points of intersection between health literacy and shared decision-making and identify new approaches to intervention which can bring us a step closer in achieving the shared goal of supporting empowerment, control, and self-efficacy that lead patients to value autonomy and enable them to participate more fully in making health decisions. Bringing these issues to the fore can help to create a shared dialog moving forward, and provide strategic direction for future research into building the skills and capacity of both providers and patients.

Acknowledgments: We would like to acknowledge Dr. Sian Smith and Associate Professor Haryana Dhillon for their comments on early drafts of this manuscript.

Corresponding Author: Danielle M. Muscat, PhD; Faculty of Medicine and Health, School of Public Health, Sydney Health Literacy Lab, The University of Sydney Sydney, NSW, Australia (e-mail: danielle.muscat@sydney.edu.au).

\section{Compliance with Ethical Standards:}

Conflict of Interest: The authors declare that they do not have a conflict of interest.

\section{REFERENCES}

1. Pleasant A. Health literacy: an opportunity to improve individual, community, and global health. New Dir Adult Cont Educ 2011;130:4353.

2. Sørensen K, Van Den Broucke S, Fullam J, et al. Health literacy and public health: a systematic review and integration of definitions and models. BMC Public Health 2012;12:80-80.

3. Nutbeam D, McGill B, Premkumar P. Improving health literacy in community populations: a review of progress. Health Promot Int 2018;33(5):901-911.

4. Nutbeam D. The evolving concept of health literacy. Soc Sci Med 2008;67(12):2072-2078.

5. Kaphingst KA, Kreuter MW, Casey C, et al. Health Literacy INDEX: development, reliability, and validity of a new tool for evaluating the health literacy demands of health information materials. $J$ Health Commun. 2012;17(sup3):203-221.

6. Nutbeam D. Health literacy as a public health goal: a challenge for contemporary health education and communication strategies into the 21st century. Health Promot Int 2000;15(3):259-267.

7. Edwards M, Wood F, Davies M, Edwards A. The development of health literacy in patients with a long-term health condition: the health literacy pathway model. BMC Public Health 2012;12(1):130-130.

8. Stiggelbout AM, Weijden TVD, Wit MPTD, et al. Shared decision making: trade-offs between narrower and broader conceptions. Health Expect 2011;14(2):210.

9. McCaffery KJ, Smith SK, Wolf M. The challenge of shared decision making among patients with lower literacy: a framework for research and development. Med Decis Mak 2010;30(1):35-44.
10. McCaffery KJ, Holmes-Rovner M, Smith SK, et al. Addressing health literacy in patient decision aids. BMC Med Inform Decis Mak 2013;13(2):S10-S10.

11. Pryce H, Durand M-A, Hall A, et al. The development of a decision aid for tinnitus. Int J Audiol 2018;57(9):714-719.

12. Smith SK, Trevena L, Simpson JM, Barratt A, Nutbeam D, McCaffery KJ. A decision aid to support informed choices about bowel cancer screening among adults with low education: randomised controlled trial. BMJ. 2010;341(7780):977-977.

13. Barton JL, Koenig CJ, Evans-Young G, et al. The design of a low literacy decision aid about rheumatoid arthritis medications developed in three languages for use during the clinical encounter. BMC Med Inform Decis MaK 2014;14(1): 104-114.

14. The Dartmouth Institute for Health Policy and Clinical Practice. Option Grid $^{\mathrm{TM}}$ decision aids. Available at: http://optiongrid.org/. Accessed February 28, 2020.

15. Smith SA. Promoting health literacy: concept, measurement and intervention. Cincinnati: Union Institute \& University; 2009.

16. Towle A, Godolphin W. Framework for teaching and learning informed shared decision making. BMJ 1999;319(7212):766-769.

17. Makoul G, Clayman ML. An integrative model of shared decision making in medical encounters. Patient Educ Couns 2006;60(3):301-312.

18. Lown BA, Clark WD, Hanson JL. Mutual influence in shared decision making: a collaborative study of patients and physicians. Health Expect 2009, 12(2): 160 .

19. Muscat DM, Shepherd HL, Nutbeam D, et al. Developing verbal health literacy with adult learners through training in shared decision making. Health Literacy Res Pract 2017;1(4):e257-e268.

20. Smith SK, Nutbeam D, McCaffery KJ. Insights into the concept and measurement of health literacy from a study of shared decision-making in a low literacy population. J Health Psychol 2013; 18(8): 1011-1022.

21. Hoffmann TC, Montori VM, Del Mar C. The connection between evidencebased medicine and shared decision making. JAMA. 2014;312(13):12951296. https://doi.org/10.1001/jama.2014.10186.

22. Charles C, Gafni A, Whelan T. Shared decision-making in the medical encounter: what does it mean? (or it takes at least two to tango). Soc Sci Med 1997:44(5):681-692.

23. England C, Morgan R. Communication skills for midwives: challenges in everyday practice. Maidenhead: McGraw-Hill Education; 2012.

24. Hambly H, Robling M, Crowne E, Hood K, Gregory JW, Team DS. Communication skills of healthcare professionals in paediatric diabetes services. Diabet Med 2009;26(5):502-509.

25. McCorry LK, Mason J. Communication skills for the healthcare professional. Philadelphia: Wolters Kluwer Health/Lippincott Williams \& Wilkins; 2011

26. Kraszewski S, McEwen A: Communication skills for adult nurses. Maidenhead: Open University Press; 2010.

27. Sheridan SL, Halpern DJ, Viera AJ, Berkman ND, Donahue KE, Crotty K. Interventions for individuals with low health literacy: a systematic review. J Health Commun 2011;16(Supp3):30-54.

28. Visscher BB, Steunenberg B, Heijmans M, Hofstede JM, Deville W, van der Heide I, Rademakers J. Evidence on the effectiveness of health literacy interventions in the EU: a systematic review. BMC Public Health. 2018;18(1).

29. Schubbe D, Cohen S, Yen RW, et al. Does pictorial health information improve health behaviours and other outcomes? A systematic review protocol. BMJ Open 2018;8(8):e023300.

30. Muscat DM, Morony, S., Hayen, A. Skills for shared decision-making: evaluation of a health literacy program for lower literacy consumers. Health Literacy Res Pract 2019;3(Suppl. 1):S58-S74

31. Nutbeam D. Defining, measuring and improving health literacy. Health Eval Promotion 2015;42: 450-55.

32. Nsangi A, Semakula D, Oxman AD, et al. Effects of the Informed Health Choices primary school intervention on the ability of children in Uganda to assess the reliability of claims about treatment effects: a clusterrandomised controlled trial. Lancet. 2017;390(10092):374-388.

33. Kaper MS, Sixsmith J, Koot JAR, et al. Developing and pilot testing a comprehensive health literacy communication training for health professionals in three European countries. Patient Educ Couns 2018;101(1):152-158.

34. Joseph-Williams N, Edwards A, Elwyn G. Power imbalance prevents shared decision making. BMJ. 2014;348(7960):17-17.

Publisher's Note: Springer Nature remains neutral with regard to jurisdictional claims in published maps and institutional affiliations. 